\title{
ISO-SWS Spectroscopy of B[e] Stars
}

\author{
Robert H.M. Voors ${ }^{1,2}$, Laurens B.F.M. Waters ${ }^{3,4}$, and Patrick W. Morris ${ }^{5,2}$ \\ 1 Astronomical Institute, Utrecht University, The Netherlands \\ ${ }^{2}$ SRON Laboratory for Space Research, Utrecht, The Netherlands \\ 3 Astronomical Institute, University of Amsterdam, The Netherlands \\ 4 SRON Laboratory for Space Research, Groningen, The Netherlands \\ 5 ISO Science Operations Center, Villafranca, Madrid, Spain
}

\begin{abstract}
We present ISO-SWS spectra of $\mathrm{B}[\mathrm{e}]$ stars. We find a wide diversity of spectral characteristics of $\mathrm{B}[\mathrm{e}]$ stars, suggesting different origins for the circumstellar matter. Most $\mathrm{B}[\mathrm{e}]$ supergiants show hot dust with weak amorphous silicate emission. MWC 300 has a warm dust shell with strong crystalline silicate emission; its evolutionary status is unclear.
\end{abstract}

\section{Introduction}

B $[\mathrm{e}]$ stars are defined by the following characteristics: (1) Spectral type B (2) Optical emission lines; hydrogen recombination and low ionisation metal lines, both permitted and forbidden (3) IR excess due to (hot) dust. The main problem with this definition is that it is purely phenomenological and does not constrain the evolutionary state of the object. This is illustrated by the fact that the above defined group of $\mathrm{B}[\mathrm{e}]$ stars contains such diverse objects as $\mathrm{B}[\mathrm{e}]$ supergiants, $\mathrm{HAeBe}$ stars, PPNe, symbiotic stars and also a group of stars of which the evolutionary state is unknown (Lamers et al. 1998). We will use spectra taken with the ShortWave Spectrometer (SWS) on board the Infrared Space Observatory (ISO) of a number of $\mathrm{B}[\mathrm{e}]$ stars, with a focus on the $\mathrm{B}[\mathrm{e}]$ supergiants, to study the region between 2.4 and $45 \mu \mathrm{m}$.

\section{$2 \quad \mathrm{~B}[\mathrm{e}]$ Supergiants}

The widely accepted model for $\mathrm{B}[\mathrm{e}]$ supergiants was first proposed by Zickgraf et al. (1985). In this model, the star has a two-component wind. In the polar region, a fast $\left(\mathrm{v}_{\text {exp }} \approx 1000 \mathrm{kms}^{-1}\right.$ ), hot (C IV, Si IV), low density wind is present, similar to normal B supergiants. In the equatorial region, both the expansion velocity $\left(\approx 100 \mathrm{kms}^{-1}\right)$ and the temperature ( $\mathrm{Si}$ II, Fe II) are much lower; assuming a constant mass flux, the density is much higher. The wind in the equatorial region is effectively shielded from UV radiation, and at some point has cooled enough and still has a sufficient density, for dust to form.

This model predicts an energy distribution which shows the central star plus some free-free excess and, superposed on that, the thermal continuum from the dusty disk. Dust condenses typically between 1000 and $1600 \mathrm{~K}$, 
which corresponds to a few $10^{3} R_{*}$. A temperature of $1300 \mathrm{~K}$ corresponds to a peak in the energy distribution $\left(F_{\nu}\right)$ at $4 \mu \mathrm{m}$. If we assume that the formation of the dust does not influence the large scale density distribution of the wind, then the dust density distribution will follow the same $r^{-2}$ distribution as the gas. So we expect a more or less normal B supergiant continuum, and superposed a thermal dust continuum that peaks roughly between 3 and $5 \mu \mathrm{m}$, with a steep decline (because of the steep density gradient) towards longer wavelengths.

This is exactly what is observed in several sources, e.g. in CPD $-57^{\circ} 2874$. The optical spectrum of this star is dominated by $\mathrm{HI}$ and He I emission lines (Carlson \& Henize 1979). McGregor et al. (1988) discovered CO first overtone emission at $2.3 \mu \mathrm{m}$, which indicates the presence of a high density region $\left(n>10^{10} \mathrm{~cm}^{-3}\right)$ around the star. Using an optically thin, spherically symmetric model to fit the SWS spectrum and assuming an outflow velocity in the dust forming region of $100 \mathrm{kms}^{-1}$, we derive a total dust mass-loss rate for CPD $-57^{\circ} 2874$ of a few $10^{-9} \mathrm{M}_{\odot} \mathrm{yr}^{-1}$. McGregor et al. derive a total mass-loss rate, based on the strength of $\operatorname{Br} \gamma$ of a few $10^{-6} \mathrm{M}_{\odot} \mathrm{yr}^{-1}$. Assuming a gas/dust ratio of 100 , which is the canonical value found in the interstellar medium, this suggests an opening angle of the disk of the order of 20 degrees. These are very rough numbers but it confirms the idea that only in the equatorial region dust is formed around $\mathrm{B}[\mathrm{e}]$ stars. It also indicates that probably this region is not extremely thin; less than 1 degree seems unlikely. Since it will be difficult to get a good constraint on the gas/dust ratio, a very tight constraint on exactly how much of the wind contains dust - and how much of it takes part in the formation process - will be difficult to determine.

\section{MWC 300: is it a supergiant?}

One of the objects with an unclear evolutionary status is MWC 300 . Wolf $\&$ Stahl (1985) conclude it must be a supergiant, primarily based on the presence of multiplets 115 and 117 of Fe III in emission in the optical spectrum. However, it is also included in a number of pre-main-sequence studies (e.g. Thé et al. 1994), even though it does not clearly show any pre-main-sequence characteristics. Henning et al. (1994) derive a mass of $300 \mathrm{M}_{\odot}$ of circumstellar dust and gas, based on their $1.3 \mathrm{~mm}$ flux $(54 \pm 15 \mathrm{mJy})$ and on the distance of $15.5 \mathrm{kpc}$, as derived by Wolf \& Stahl (1985). Even though there is a very large uncertainty in this mass (they estimate at least a factor of 5), it seems too large to be explained as a post-main-sequence shell.

The SWS spectrum of MWC 300 is shown in figure 1a. The dust in MWC 300 shows a very wide range in temperatures and is much cooler than that of more "typical" B[e] supergiants, such as CPD $-57^{\circ} 2874$. This suggests a flat density gradient and/or significant optical depth effects. The $9.7 \mu \mathrm{m}$ amorphous silicate feature is seen in absorption, and this may very well be of interstellar origin. The SWS spectrum of MWC 300 shows prominent narrow 
emission bands that can be attributed to $\mathrm{Mg}$-rich, Fe-poor crystalline olivines $(19.5,23.5,27.5$ and $33.5 \mu \mathrm{m})$ and pyroxenes (most of the narrower peaks). These dust components are often observed in cool stars with very dense, dusty outflows (e.g. red supergiants, $\mathrm{OH} / \mathrm{IR}$ stars), and also in objects with circumstellar or circum-binary disks (e.g. HAeBe stars).

The origin of the dust is not clear. It is unlikely that it is formed in a present day outflow, because (1) the slope of the SED does not agree with an $r^{-2}$ density distribution, and (2) the dust-forming layers are at leare distance from the (hot) star, where the density is low; crystalline silicates are only observed in objects with very high density in the dust-forming layers. Another possibility is a non-outflow disk. This alleviates the above mentioned difficulties, but the origin of such a disk in unclear. From low mass stars it is observed that disks containing crystalline dust are often associated with binaries.
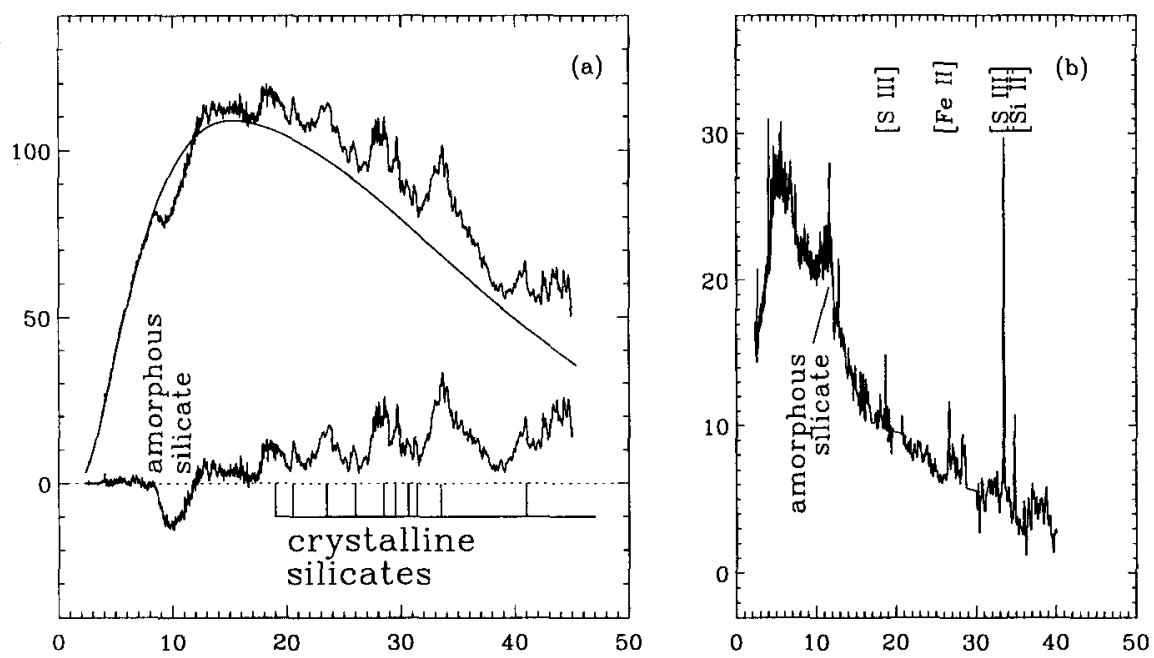

Fig. 1. SWS spectra of MWC 300 (a) and CPD $-57^{\circ} 2874$ (b). The lower spectrum of MWC 300 is continuum (drawn line) subtracted. Abscissa: wavelength in $\mu \mathrm{m}$; Ordinate: flux density in Jansky

\section{References}

Carlson E.D., Henize K.G., 1979, Vistas Ast. 23, 213

Henning Th., Launhardt R., Steinacker J., Thaumm E., 1994, A\&A 291, 546

Jäger C., Molster F.J., Dorschner J., et al., 1998, submitted to A\&A

Lamers H.J.G.L.M., Zickgraf F.-J., de Winter D., et al., 1998, submitted to A\&A

McGregor P.J., Hyland A.R., Hillier D.J., 1988, ApJ 324, 1071

Thé P.S., de Winter D., Perez M.R., 1994, A\&AS 104, 315

Wolf B., Stahl B., 1985, A\&A 148, 412

Zickgraf F.-J., Wolf B., Stahl O., et al., 1985, A\&A 143, 421 


\section{Discussion}

S. Shore: A question and a comment: Do the B supergiants (or B[e] stars) that show CO $2 \mu$ emission show the PAH features? Since HD 87643 is also known as He 3-365, I should mention that this star shows variations in the $\mathrm{UV}$ of $\sim 30 \%$ on timescales of about a decade. It has one of the very strongest UV iron curtain absorption spectra of any LBV.

R. Voors: Stars in our sample that are known to show CO emission in the $\mathrm{K}$-band do not show PAH features.

M. Magalhães: Was ISO sensitive enough to observe $\mathrm{B}[\mathrm{e}]$ supergiants in the Magellanic Clouds?

R. Voors: Some B $[\mathrm{e}]$ supergiants in the LMC/SMC were observed with the PHOT-S instrument onboard ISO $(\lambda / \Delta \lambda \sim 100)$, but they are too faint to be observed with the Short Wave Spectrometer.

P. Williams: Considering the unusual density distribution in the disk, have you looked at the infrared photometric history? I have ground-based $11 \mu \mathrm{m}$ observations from 20 years ago.

R. Voors: The ISO SWS fluxes are consistent with the IRAS fluxes. But I would certainly be interested to see your even older data of the silicate emission bump.

F.-J. Zickgraf: Could you see any $10 \mu \mathrm{m}$ silicate feature in Magellanic Cloud $\mathrm{B}[\mathrm{e}]$ supergiants?

R. Voors: Maybe, I do not know.

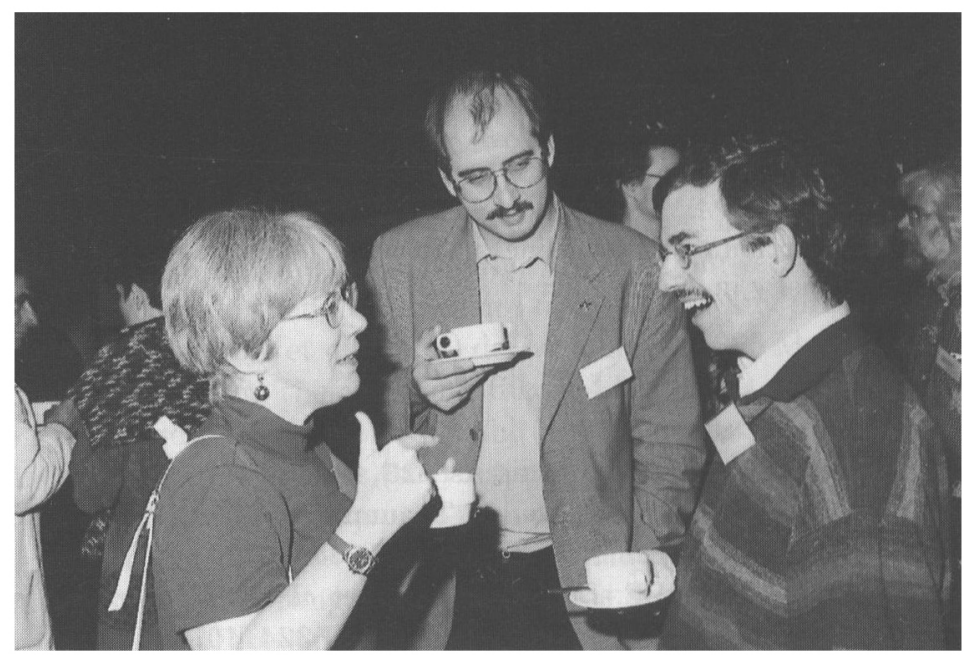

Roberta Humphreys, Norman Trams and Rens Waters 